\title{
Free Quotients of Finitely Presented Groups
}

\author{
Derek F. Holt and Sarah Rees
}

\section{CONTENTS}

1. Introduction

2. Application

3. A Question About the Picard Group

4. Searching for Additional Relations

5. The Picard Group Computations

References
We describe a computational, heuristic approach to the problem of deciding whether or not a given finitely presented group has a free quotient of rank two or more. Our strategy is to construct a finite nilpotent quotient of the given group, to search for quotients that are free within a variety containing that quotient, and then lift to the original group. We give theoretical justification to our strategy, and describe successful computations with sections of the Picard group $\mathrm{SL}_{2}(\mathbb{Z}[i])$.

\section{INTRODUCTION}

This article discusses the following problem: If $G$ is a group with a given finite presentation $\langle X \mid R\rangle=$ $\left\langle x_{1}, x_{2}, \ldots, x_{m} \mid r_{1}, r_{2}, \ldots, r_{n}\right\rangle$, how can we decide whether or not $G$ has a free nonabelian quotient? Alternatively, and equivalently, how do we decide whether or not $G$ has a free quotient of rank two? We describe a computational approach to the problem, relating to actual calculations with the Picard group $\mathrm{SL}_{2}(\mathbb{Z}[i])$.

We know that $G$ has a free quotient of rank $k$ if and only if there is a homomorphism from the free group on $X$ onto the free group of rank $k$ whose kernel contains each of the words in $R$. Such homomorphisms correspond to sequences $\left(w_{1}, \ldots, w_{m}\right)$ of freely reduced words in $k$ generators (where $w_{i}$ is the image of $x_{i}$ ), and can be enumerated by a backtrack search. That a given homomorphism is surjective can also be verified using a backtracking search. Thus there is a clear procedure to find eventually a free quotient if one exists. However this method can not be used to prove the nonexistence of a free quotient, and is totally impractical for large presentations. Hence we take an entirely different and heuristic approach.

We attempt to construct free quotients of $G$ by identifying additional relations to add to those in 
the given presentation, that is, we search for a set $R^{\prime}$ of words in the generators for $G$ such that the group $\left\langle X \mid R \cup R^{\prime}\right\rangle$ is free.

Briefly our strategy is as follows. We start by looking not at $G$ but at a nilpotent quotient $\Gamma$ of $G$. Typically $\Gamma$ is in the variety of $p$-groups, for some prime $p$, with exponent $p$ class 2 ; occasionally we need to look at a higher class. We search for additional relations that should be added to define a quotient of $\Gamma$ that is free within a restricted variety of groups containing $\Gamma$. If we are successful in our search, we lift those additional relations to $G$, and hope that the quotient of $G$ thus defined is free (we verify this by simplifying the presentation we now have with Tietze transformations). If it is not we try again with a larger quotient $\Gamma$.

The remainder of this paper is organised as follows. Section 2 describes our motivation for examining the problem, and discusses other possible applications. Section 3 gives some details about the particular problem with the Picard group that we were trying to solve. Section 4 describes in detail the strategy outlined above, and gives some theoretical justification. Section 5 describes our successful calculations with the Picard group.

\section{APPLICATION}

Our original interest in this problem arose out of a problem from Alec Mason about the principal congruence subgroups of the Picard group $\mathrm{SL}_{2}(\mathbb{Z}[i])$. Details will given in Section 3, and we shall use this example to illustrate our approach to searching for free quotients, which will be described in Section 4 . But the question appears to be of more general interest. Certainly a group with a nonabelian free quotient has a very rich finite quotient structure, and maps onto any finite simple group. Clearly also such a group is SQ-universal, that is, has every countable group as a subgroup of a quotient.

Knowledge of free quotients may also be interesting for three-manifold groups, since it gives information about the possible decompositions of a group as an HNN-extension or a free product with amalgamation. For example, by [Dicks and Dunwoody 1989, Theorem V1.4.5], if a finitely presented group $G$ has a free quotient of rank $n$, it is of the form $A *_{B}$ where $A$ and $B$, are finitely generated and $A$ has a free quotient of rank $n-1$. (Recall that $A *_{B}=\langle A, t| t b t^{-1}=\varphi(b)$ for $\left.b \in B\right\rangle$, where $\varphi$ is an isomorphism from the subgroup $B$ of $A$ to a subgroup $C$ of $A$.)

Waldhausen's conjecture (raised as a problem in [Waldhausen 1978]) states that every irreducible three-manifold $M$ has a finite cover $M^{\prime}$ that is either simply connected or Haken (admits an embedding of a closed surface $S$ whose fundamental group embeds nontrivially in that of $M^{\prime}$, and hence which allows a decomposition of $M^{\prime}$ ). If $M^{\prime}$ is Haken, its fundamental group is either a free product with amalgamation (if $S$ separates $M^{\prime}$ into two disjoint manifolds) or an HNN-extension. A stronger form of Waldhausen's conjecture states that $M$ must always have a finite cover whose fundamental group is either trivial or an HNN-extension.

An algorithm to search directly for decompositions of a given finitely presented group as an HNN-extension or free product with amalgamation is described in [Bartholomew 1987], and is implemented as a C-program to run on DOS machines. The algorithm involves the construction of a two-dimensional cell complex from the presentation, and then a search for 'tracks' (certain subsets of the complex) that give information about possible decompositions. In general there are infinitely many tracks, but for certain presentations a finite set of tracks is sufficient to determine the existence of a decomposition, so the algorithm decides conclusively whether of not the group decomposes.

\section{A QUESTION ABOUT THE PICARD GROUP}

Let $G$ be the Picard group $\mathrm{SL}_{2}(\mathbb{Z}[i])$. For any integer $n$, let $G(n)$ be the kernel of the natural map from $G$ to $\left.\mathrm{SL}_{2}(\mathbb{Z}[i] / n \mathbb{Z}[i])\right)$, and let $E(n)$ be the normal closure in $G$ of the subgroup generated by the $n$-th powers of the matrices $u=\left(\begin{array}{ll}1 & i \\ 0 & 1\end{array}\right)$ and 
$t=\left(\begin{array}{ll}1 & 1 \\ 0 & 1\end{array}\right)$. Finally, let $\operatorname{GE}(n)$ be the quotient of $G(n) / E(n)$.

In a private communication, Alec Mason asked if one could find out for which $n$ the group $\operatorname{GE}(n)$ has a free nonabelian quotient. He conjectured that there was such a quotient for all but finitely many $n$. If there were free nonabelian quotients for some $n$, then the Picard group would have uncountably many nonnormal 'standard' subgroups.

Our calculations showed that $\operatorname{GE}(5), \operatorname{GE}(6)$ and $\mathrm{GE}(7)$ had free quotients of rank two. The calculations, of which some details are given below, were large and so caused us to develop what appears to be an effective strategy to deal with the problem. It would probably be possible to carry out similar calculations in $\operatorname{GE}(n)$ for larger values of $n$, although this starts to become more difficult as the index of $\mathrm{GE}(n)$ in $G$ increases, because the length of the computed presentation for $\operatorname{GE}(n)$ increases roughly in proportion to this index.

To put this particular problem into perspective, we remark that Bass, Milnor and Serre proved in [Bass et al. 1967] that, for any arithmetic Dedekind domain $A$, no subgroup of finite index in $\mathrm{SL}_{n}(A)$ has a free nonabelian quotient when $n>2$. This is also true for $n=2$ when $A$ has infinitely many units, so the interesting groups from this point of view are $\mathrm{SL}_{2}(A)$ where $A$ has finitely many units.

Let $d$ be a positive integer and let $R=\mathcal{O}_{d}$ be the ring of integers of $\mathbb{Q}[\sqrt{ }-d]$. Mason and Odoni [1996] have now proved without the use of computers that, for infinitely many ideals $I$ of $R$, the kernel $K$ of the surjection $\mathrm{SL}_{2}(R) \rightarrow \mathrm{SL}_{2}(R / I)$ has a free nonabelian quotient whose kernel contains all the elementary matrices of $K$. Hence it is now known that every Bianchi group $\mathrm{SL}_{2}(R)$ has uncountably many nonnormal 'standard' subgroups. Another related result, proved recently by Grunewald, Mennicke, and Vaserstein, is that $\mathrm{SL}_{2}(\mathbb{Z}[t]) / \mathrm{U}_{2}(\mathbb{Z}[t])$ has free quotients of arbitrarily large ranks.

The theoretical methods used to prove results of this sort are only useful for congruence subgroups of large index, so the use of the computer is likely to remain important for examining low-index cases.
We tried to perform corresponding calculations in some of the other Bianchi groups, and in particular for $d=2$ and 3 . We were in fact successful in finding free quotients of the corresponding groups GE(5) in these two cases. Since these calculations did not contain any distinctive features, however, we shall restrict ourselves here to describing the case $d=1$, the Picard group.

The following presentation for $\mathrm{SL}_{2}(\mathbb{Z}[i])$ is given in [Swan 1968]:

$$
\begin{aligned}
& \langle a, t, u, l, j| \\
& \quad a^{2}=l^{2}=(t a)^{3}=(a l)^{2}=(t l)^{2}=(u l)^{2}=(u a l)^{3}=j, \\
& \left.j^{2}=[a, j]=[t, j]=[u, j]=[l, j]=[t, u]=1\right\rangle .
\end{aligned}
$$

The elements $a, t, u, l, j$ can be represented by the following $2 \times 2$ matrices over the Gaussian integers:

$$
\begin{aligned}
& a=\left(\begin{array}{rr}
0 & -1 \\
1 & 0
\end{array}\right), t=\left(\begin{array}{ll}
1 & 1 \\
0 & 1
\end{array}\right), u=\left(\begin{array}{ll}
1 & i \\
0 & 1
\end{array}\right) \\
& l=\left(\begin{array}{rr}
-i & 0 \\
0 & i
\end{array}\right), j=\left(\begin{array}{rr}
-1 & 0 \\
0 & -1
\end{array}\right) .
\end{aligned}
$$

We used standard computational techniques to build presentations for the groups $\operatorname{GE}(n)$ for $n \leq 6$. More precisely, for a particular $n$, we started by adjoining the relations $t^{n}=u^{n}=1$ to those of $G=\mathrm{SL}_{2}(\mathbb{Z}[i])$ to give us a presentation of $G / E(n)$.

We used our program QUOTPIC [Holt and Rees 1993] to obtain, via descending series with simple and elementary abelian factors, presentations for normal subgroups of $G / E(n)$, and generators for those subgroups, as words in the original generators for $G / E(n)$. The matrices representing the subgroup generators could easily be computed as products of the matrices listed above (an elementary task, using GAP [Schönert et al. 1994]), allowing us to identify $\mathrm{GE}(n)$ precisely as the kernel of the map of $G / E(n)$ onto $\mathrm{SL}_{2}(\mathbb{Z}[i] / n \mathbb{Z}[i]) / E(n)$ from among various normal subgroups at the bases of series with the same composition factors as

$$
\mathrm{SL}_{2}(\mathbb{Z}[i] / n \mathbb{Z}[i]) / E(n) .
$$


(Where $n=\xi_{1}^{k_{1}} \xi_{2}^{k_{2}} \ldots \xi_{r}^{k_{r}}$ as a product of Gaussian primes, the group $\mathrm{SL}_{2}(\mathbb{Z}[i] / n \mathbb{Z}[i])$ is a product of factors

$$
\mathrm{SL}_{2}\left(\mathbb{Z}[i] / \xi_{i}^{k_{i}} \mathbb{Z}[i]\right)
$$

Each of these groups has a normal series with top factor $\mathrm{SL}_{2}\left(\mathbb{Z}[i] / \xi_{i} \mathbb{Z}[i]\right) \cong \mathrm{SL}_{2}(q)$, where $q=\left|\xi_{i}\right|^{2}$, and $k-1$ other factors each isomorphic to the additive group of $2 \times 2$ trace-zero matrices over $G F(q)$.)

In fact the indices of $\operatorname{GE}(n)$ in $G / E(n)$ for $n=$ $2,3,4,5,6$ are $48,720,3072,14400$ and 34560 , and in each case we used the Reidemeister-Schreier algorithm to obtain a presentation of this subgroup.

It is not difficult to prove directly from these presentations that $\mathrm{GE}(2)$ and $\mathrm{GE}(3)$ are trivial, and that $\mathrm{GE}(4)$ is free abelian of rank 4. Using the methods of this paper we were able to show that each of $\mathrm{GE}(5), \mathrm{GE}(6)$ and $\mathrm{GE}(7)$ has a free quotient of rank two. In all three cases the presentations were large. For GE(5), we obtained, after simplification, a presentation with 24 generators and 115 relators, and total length 4598 (since the derived quotient is free abelian of rank 24, there can be no presentation with fewer than 24 generators). For $\mathrm{GE}(6)$ we found a presentation with 209 generators and 585 relators, and total length 12985. The index of $\operatorname{GE}(7)$ in $G / E(7)$ is 117600 (the factor group is $\mathrm{SL}(2,49)$ ), and although it was still possible to compute a subgroup presentation of $\mathrm{GE}(7)$, the result was too long and unwieldy to be useful for further calculations. We therefore tried to find a subgroup $H / E(7)$ of $G / E(7)$, properly containing $\mathrm{GE}(7)$, for which we could find a free quotient of rank two or more. Since, by the Schreier subgroup theorem [Hall 1959, Theorem 7.2.8], a subgroup of index $n$ in a free group of rank $r$ is free of rank $1+n(r-1), \operatorname{GE}(7)$ itself must have a free quotient of rank at least two. After some experimentation, we found such a subgroup of index 1225 (the stabiliser of an unordered pair in the natural permutation representation of $\mathrm{SL}(2,49))$. This presentation initially had 409 generators, 768 relators and length 2378 .
Details of how we found the free quotients of each of these three groups are given in Section 5 .

\section{SEARCHING FOR ADDITIONAL RELATIONS}

We search for a set $R^{\prime}$ of words in the generators for $G$ such that the group $\left\langle g_{1}, \ldots, g_{m} \mid R \cup R^{\prime}\right\rangle$ is free, that is, we look for relations we can add to $G$ to make it free.

We look first not at $G$ but at a quotient $\Gamma$ of $G$, and search for a quotient of $\Gamma$, which is free within the variety containing $\Gamma$. The rank of that quotient of $\Gamma$ bounds the rank of a free quotient of $G$. Then we try to lift the new relations we have added to $\Gamma$ up to $G$.

We need only find a good choice for $\Gamma$. Clearly $\Gamma$ must be large enough that quotients of $\Gamma$ that are free within its variety have a reasonable chance of lifting to free quotients of $G$. It must also be small enough for the computation of its presentation to be feasible. (In practice our presentation for $G$ may be very large.)

We elect to choose $\Gamma$ from among various abelian and nilpotent quotients of $G$, namely the maximal abelian quotient $G / G^{\prime}$ of $G$, the maximal elementary $p$-abelian quotient $G / G^{\prime} G^{p}$ of $G$, the maximal nilpotent quotient $G / \gamma_{c+1}(G)$ of class $c$ (defined by $\gamma_{1}(G)=G$ and $\left.\gamma_{i+1}(G)=\left[\gamma_{i}(G), G\right]\right)$, and the maximal nilpotent $p$-quotient $G / \gamma_{c+1, p}(G)$ of exponent $p$ class $c$ (defined by $\gamma_{1, p}(G)=G$, $\gamma_{2, p}(G)=G^{\prime} G^{p}$, and $\left.\gamma_{i+1, p}(G)=\left[\gamma_{i, p}(G), G\right] G^{p}\right)$. For all of these choices, presentations in standard form can be computed relatively easily by solving systems of linear equations over the integers (or integers $\bmod p$, for some prime $p$ ). An abelian quotient of a group is maybe too small a slice of it to be of much use here, and $\bmod p$ calculations are often feasible where integer calculations are not; hence a good choice seems to be to choose $\Gamma$ to be $G / \gamma_{3, p}(G)$ for some $p$.

Our strategy is as follows:

Step 1. (a) Add relations $w=1$ to $G$ for relations $w^{k}=1$ for $k>1$, and for any word $w$ that is clearly implied by the presentation to be central. 
(b) Add relations $g_{i}=1$ for all generators appearing in short relators of exponent sum 0.

In this way we form a quotient $G_{0}$ of $G$ with a smaller presentation. We search for a free quotient of $G_{0}$.

Step 2. Compute the $p$ group $\Gamma=G_{0} / \gamma_{3, p}\left(G_{0}\right)$ for some prime $p$.

Step 3. Find relations that define a quotient of $\Gamma$ that is free in the variety $\mathcal{N}_{2, p}$ of $p$-groups with exponent $p$ class 2 .

Step 4. Lift the new relations to $G$ and hope to find a free quotient of $G$. If not, replace $G_{0}$ by the current quotient and repeat steps 2 and 3 with $\Gamma=G_{0} / \gamma_{c+1, p}\left(G_{0}\right)$ for some $c>2$.

Tietze transformations are used to simplify the presentations at each stage after new relations have been added; we know that we have a free quotient when the presentation simplifies to one with no relations!

Step 1 is a preprocessing step, to help us to handle large presentations. Since free groups have no torsion or centre, central and torsion elements must lie in the kernel of any free quotient, so nothing can be lost by performing step 1(a). In step 1(b), we choose to kill off generators in relators of exponent sum 0 because we are interested in causing the collapse of commutator relations in $G$. Of course, not all such generators are necessarily in the kernel of a free quotient, but this seems a reasonable tactic if the presentation would otherwise be unmanageable. Precisely what is meant by a short relator may well be decided by trial and error. Step 2 can be carried out using the ANU $p$-quotient algorithm (described in [Havas and Newman 1980; Newman and O'Brien]). Step 3 is explained below. For step 4 , we rely on good luck and intuition.

It turns out that a finitely presented group $\Gamma$ in $\mathcal{N}_{2, p}$ has a quotient that is free in $\mathcal{N}_{2, p}$ if and only if a particular system of second-order equations has a solution from the integer set $\{0, \ldots, p-1\}$, which we describe below. A similar result is true for higher nilpotency classes; for $\mathcal{N}_{c, p}$ the equations are of order $c$.
The ANU $p$-quotient algorithm computes a standard power commutator presentation for the group $\Gamma=G / \gamma_{3, p}(G)$. This is a presentation on generators

$$
g_{1}, \ldots, g_{m_{1}}, g_{m_{1}+1}, \ldots, g_{m_{1}+m_{2}},
$$

of which the first $m_{1}$ are known as the weight-one generators and the last $m_{2}$ as the weight-two generators, with relations as follows:

(i) for each generator $g_{i}$ with $i>m_{1}$, relations $g_{i}^{p}=$ 1 and $\left[g_{j}, g_{i}\right]=1$ for each $j<i$,

(ii) for each generator $g_{i}$ with $i \leq m_{1}$, relations

$$
\begin{aligned}
g_{i}^{p} & =w\left(g_{m_{1}+1}, \ldots, g_{m_{1}+m_{2}}\right), \\
{\left[g_{j}, g_{i}\right] } & =w^{\prime}\left(g_{m_{1}+1}, \ldots, g_{m_{1}+m_{2}}\right) \quad \text { for } j<i .
\end{aligned}
$$

A standard power commutator presentation has the additional property that, for each weight-two generator $g_{k}$, one of the relations is designated as the definition of $g_{k}$, and has right-hand side equal to $g_{k}$. (The class- $c$ quotient $G / \gamma_{c+1, p}(G)$ has a similar presentation, with generators of weights 1,2 , $\ldots, c$.)

From now on we suppose that $\Gamma \in \mathcal{N}_{2, p}$ is given by a standard power commutator presentation as described above. The following result is straightforward to prove.

Lemma 4.1. Let $\Gamma / N$ be free in $\mathcal{N}_{2, p}$. Then there are weight-one generators $g_{i_{1}}, \ldots, g_{i_{f}}$ such that the elements $N g_{i_{1}}, \ldots, N g_{i_{f}}$ freely generate $\Gamma / N$ in $\mathcal{N}_{2, p}$. In particular:

(i) Set $w_{r s}=\left[g_{i_{r}}, g_{i_{s}}\right]$ and $w_{r 0}=g_{i_{r}}^{p}$. Then $\left\langle N w_{r s}\right\rangle$ is elementary $p$-abelian of rank $\frac{1}{2} f(f+1)$.

(ii) Each relation in $\Gamma / N$ is a consequence of the $N w_{r s}$ 's being central in $\Gamma / N$ of order $p$.

Conversely, suppose that $g_{i_{1}}, \ldots, g_{i_{f}}$ are weight-one generators for $\Gamma$, and for each remaining weightone generator $g_{i}$ let $u_{i}$ be a product $v_{i} g_{i}^{-1}$, where $v_{i}$ is a word in $g_{i_{1}}, \ldots, g_{i_{f}}$, and let $N=\left\langle u_{i}\right\rangle^{\Gamma}$. Then $\Gamma / N$ is generated by $\left\{N g_{i_{r}}\right\}, \Gamma / N$ is free in $\mathcal{N}_{2, p}$ if and only if (i) and (ii) hold. 
We use this lemma to derive a set of second-order equations whose solution is equivalent to the existence of a quotient $\Gamma / N$ as above. We introduce some simplifying notation to make the equations more readable. If $a=\left(a_{j r}\right)$ is an $m_{1} \times f$ integer matrix, we define $h_{r s}^{i}(a)$, where $r=1, \ldots, f$, $s=0, \ldots, f$, and $i=m_{1}+1, \ldots, m_{1}+m_{2}$, by the following rule: If the weight-two generator $g_{i}$ is defined by $\left[g_{j}, g_{k}\right]=g_{i}$, then

$$
h_{r s}^{i}(a)= \begin{cases}a_{j r} a_{k s}-a_{j s} a_{k r} & \text { if } s>0, \\ 0 & \text { if } s=0 .\end{cases}
$$

If $g_{i}$ is defined by $g_{j}^{p}=g_{i}$, then

$$
h_{r s}^{i}(a)= \begin{cases}0 & \text { if } s>0 \\ a_{j r} & \text { if } s=0 .\end{cases}
$$

Theorem 4.2. Let $\Gamma$ be a group in $\mathcal{N}_{2, p}$ given by a power commutator presentation as above. Let $g_{i_{1}}$, $\ldots, g_{i_{f}}$ be certain weight-one generators of $\Gamma$. For each weight-one generator $g_{i}$ not in $\left\{g_{i_{1}}, \ldots, g_{i_{f}}\right\}$ select $a_{i r} \in\{0, \ldots, p-1\}$ and $z_{i} \in \Gamma^{\prime} \Gamma^{p}$, and let

$$
u_{i}=\prod_{r=1}^{f} g_{i_{r}}^{a_{i r}} z_{i} g_{i}^{-1} .
$$

For $s=1, \ldots, f$, define $a_{i_{s} r}$ to be 1 if $r=s$ and 0 otherwise.

Let $N=\left\langle u_{i}\right\rangle^{\Gamma}$. Then $\Gamma / N$ is free of rank $f$ in $\mathcal{N}_{2, p}$ if and only if

(i) the vectors $\left(n_{m_{1}+1}, \ldots, n_{m_{1}+m_{2}}\right)$ of integers associated with the elements $w_{r s}$ (as defined in Lemma 4.1) are linearly independent $\bmod p$, and

(ii) the integers $a_{\text {ir }}$ satisfy

$$
\sum_{i=m_{1}+1}^{m_{1}+m_{2}} n_{i} h_{r s}^{i}(a)= \begin{cases}a_{j r} a_{k s}-a_{j s} a_{k r} & \text { if } s>0 \\ 0 & \text { if } s=0\end{cases}
$$

for each nondefining relation $\left[g_{j}, g_{k}\right]=\prod_{i=m_{1}+1}^{m_{1}+m_{2}} g_{i}^{n_{i}}$,
and

$$
\sum_{i=m_{1}+1}^{m_{1}+m_{2}} n_{i} h_{r s}^{i}(a)= \begin{cases}0 & \text { if } s>0 \\ a_{j r} & \text { if } s=0\end{cases}
$$

for each nondefining relation $g_{j}^{p}=\prod_{i=m_{1}+1}^{m_{1}+m_{2}} g_{i}^{n_{i}}$.
Proof. This follows directly from the lemma. The equations come from rewriting the left- and righthand side of each of the relations as a product of powers of the elements $w_{r s}$, after expressing each weight-one generator $g_{i}$ as a product of the form

$$
\prod_{r=1}^{f} g_{i_{r}}^{a_{i r}} z_{i}
$$

The two sides of a defining equation clearly rewrite to give the same expressions: For a nondefining relation, the two sides rewrite to the same word if and only if the relevant equation holds. Note that the choice of the elements $z_{i}$ does not affect whether or not conditions (i) and (ii) hold.

Observing that any word in weight-one generators $g_{i_{1}}, \ldots, g_{i_{f}}$ of $\Gamma$ is equal in $\Gamma$ to a word of the form $\prod_{r=1}^{f} g_{i_{r}}^{a_{i r}} z_{i}$, it is not hard to see that if $\Gamma / N$ is any free quotient then $N$ can be generated as in Theorem 4.2. Therefore:

Corollary 4.3. A group in $\mathcal{N}_{2, p}$ has a quotient that is free in $\mathcal{N}_{2, p}$ if and only if a set of weight-one generators can be selected for which the equations of Theorem 4.2 have a solution.

\section{THE PICARD GROUP COMPUTATIONS}

The results above give theoretical justification to our strategy. We finish by describing how in practice we found free quotients of the groups $\mathrm{GE}(5)$, GE(6) and GE(7), described in Section 2. Recall that, in the latter case, we did not work with $\mathrm{GE}(7)$ itself, but with an overgroup $H / E(7)$. Our aim was merely to find free quotients of rank at least two, not to find such quotients of the highest rank possible.

For GE(5) and GE(6) we first simplified the presentation for the group by adjoining relations $x_{i}=$ 1 for generators $x_{i}$ that appeared in short relators of exponent sum 0 . We did this until the class-two 5 -quotient (as computed by the ANU $p$-quotient algorithm) had a relatively straightforward presentation. At that stage, we did not attempt to solve the full system of equations of degree two derived from 
Theorem 4.2. Instead we selected two rank-one generators $g_{i_{1}}$ and $g_{i_{2}}$ in the class-two 5-quotient for which condition (i) was valid, and eliminated the remaining rank-one generators one at a time, in such a way that condition (i) continued to hold. We were able to perform the necessary calculations for this by hand; in fact almost all of the additional relations that we imposed were of form $x_{i}=1$ or $x_{i}=x_{j}^{ \pm 1}$ (where $x_{i}, x_{j}$ were generators for the original presentation), which mapped to relations between weight-one generators of the 5-quotient. Following this strategy, we successfully obtained a quotient that was free on two generators in $\mathcal{N}_{2,5}$. For $\mathrm{GE}(6)$ those relations were not enough to make the full quotient of $\mathrm{GE}(6)$ free, so we added more relations until the class-six exponent 5-quotient was also free, and then one further relation. Thus we were following the method outlined above, though in a somewhat experimental fashion, which did not involve explicit derivation and solution of the equations described (in the class-two case) by the theorem.

For GE(5) we had a presentation on 24 generators $x_{1}, \ldots, x_{24}$, with 115 relators. First we added relations of the form $x_{i}=1$ for 14 of those generators $x_{i}$, namely those that appeared in relators of length at most 14 with exponent sum zero. Then we calculated the class-two 5-quotient using the ANU $p$-quotient algorithm, and added four more relations of the form $x_{i}=1$, for those $x_{i}$ whose images were central of weight one in that quotient. Adding three more relations $x_{i}=1$ and one of the form $x_{i}=x_{j}$ finally gave us the free quotient we sought in $\mathcal{N}_{2,5}$ and a free quotient on two generators of $\mathrm{GE}(5)$.

For $\mathrm{GE}(6)$ we had a presentation on 209 generators $x_{1}, \ldots, x_{209}$, with 585 relators, and started by adding 60 relations $x_{i}=1$ for those $x_{i}$ appearing in relators of length at most 10 with exponent sum zero. Adding three more relations $x_{i}=1$ and one of the form $x_{i}=x_{j}$ made the class-two exponent 5-quotient free on two generators in $\mathcal{N}_{2,5}$, but the full quotient of $\mathrm{GE}(6)$ was not free. Next we added relations that set equal to the identity various short words (almost all of length two) that mapped to the identity of the class two 5-quotient. Now the class-six 5 -quotient was free in $\mathcal{N}_{6,5}$. Tietze transformations simplified the presentation for the quotient now defined to one with three relations of length three (amongst others). Setting the generators in three of those relators equal to the identity finally gave a free quotient of $\mathrm{GE}(6)$ on two generators.

The case of GE(7) was the easiest, once we had found a good overgroup $H / E(7)$ to work with. The presentation of this group simplified to one having length 29002, with 53 relators and 17 generators, of which seven visibly had order two. We put these equal to 1 immediately, and the presentation simplified to one with eight generators and five short relators, from which it was easy to find a free quotient of rank two, using the class-two 5-quotients as above, simply by setting some generators equal to the identity.

\section{REFERENCES}

[Bartholomew 1987] Andrew Bartholomew, "Proper decompositions of finitely presented groups", Ph.D. thesis, University of Sussex, 1987.

[Bass et al. 1967] H. Bass, J. Milnor and J.-P. Serre, "Solution of the congruence subgroup problem for $\mathrm{SL}_{n}(n \geq 3)$ and $S p_{2 n}(n \geq 2)$ ", Publ. Math. IHES 33 (1967), 59-137.

[Dicks and Dunwoody 1989] Warren Dicks and M. J. Dunwoody, Groups acting on graphs, Cambridge Studies Adv. Math. 17, Cambridge Univ. Press, Cambridge, 1989.

[Havas and Newman 1980] G. Havas and M. F. Newman, "Applications of computers to questions like those of Burnside", pp. 211-230 in Burnside Groups, Bielefeld, 1977 (edited by J. Mennicke), Lecture Notes in Math. 806, Springer, Berlin, 1980.

[Holt and Rees 1993] D. F. Holt and Sarah Rees, "A graphics system for displaying finite quotients of finitely presented groups", pp. 113-126 in Groups and Computation, New Brunswick, 1991 (edited by L. Finkelstein and W. M. Kantor), DIMACS Series 
on Discrete Math. and Theoretical Computer Science 11, Amer. Math. Soc., Providence, 1993.

[Hall 1959] Marshall Hall Jr., The Theory of Groups, Macmillan, New York, 1959. Reprinted by Chelsea, Bronx, NY, 1976.

[Mason and Odoni 1996] A. W. Mason and R. W. K. Odoni, "Non-normal, standard subgroups of the Bianchi groups", to appear in Proc. Amer. Math. Soc.

[Newman and O'Brien] M. F. Newman and E. A. O'Brien, "Applications of computers to questions like those of Burnside II", to appear in International J. Algebra Comp.
[Schönert et al. 1994] M. Schönert et al., GAP: Groups, Algorithms, and Programming, Lehrstuhl D für Mathematik, RWTH Aachen, Germany, 1994. Available by anonymous $\mathrm{ftp}$, together with the GAP system, on the servers ftp.mth.pdx.edu, archives. math.utk.edu, or math.rwth-aachen.de.

[Swan 1968] Richard G Swan, "Generators and relations for certain special linear groups", Bull. Amer. Math. Soc. 74 (1968), 576-581.

[Waldhausen 1978] Friedrich Waldhausen, "Some problems on 3-manifolds", pp. 313-322 in Algebraic and Geometric Topology, Part 2, Stanford, 1976, Proc. Symp. Pure Math. 32, Amer. Math. Soc., Providence, 1978.

Derek F. Holt, Mathematics Institute, University of Warwick, Coventry CV47AL, United Kingdom (dfh@maths.warwick.ac.uk)

Sarah Rees, Department of Mathematics and Statistics, University of Newcastle, Newcastle NE1 7RU, United Kingdom (Sarah.Rees@ncl.ac.uk)

Received July 5, 1995; accepted November 8 